\title{
eleckión del tipo de vehículo apropiado para longitudes medias de transporte
}

M. CHINCHILLA

\section{simopsis}

Para la acertada elección del tipo más apto de maquinaria para estos trabajos, habrá que tener en cuenta: Unas caracteristicas economicas, mecanicas y tecnicas que proporcionen la información necesaria para adoptar la solu-

Entre las características económicas hay que considerar los costes de adquisición, mantenimiento y entretenimiento, así como los rendimientos que se sicion, mantenimiento y entretenimiento, así como
obtendrán con el empleo de cada tipo de vehículo.

Entre las características mecánicas se consideran las que presentan cada uno de los tipos de vehículos, camiones, dumpers y camiones especiales para elegir dentro de las diversas clases y las disponibilidades existentes, aquel que por sus cualidades permita la normal realización del trabajo a efectuar, así como las condiciones mecánicas que deben satisfacer los vehículos adaptados para poder estar capacitados para competir aun en condiciones menos ventajosas con aquellos que esper
para trabajos de esta indole.

Las características técnicas que habrá que tener en cuenta para efectuar la elección son, en realidad, derivadas de las dos anteriores y dependientes de las condicion

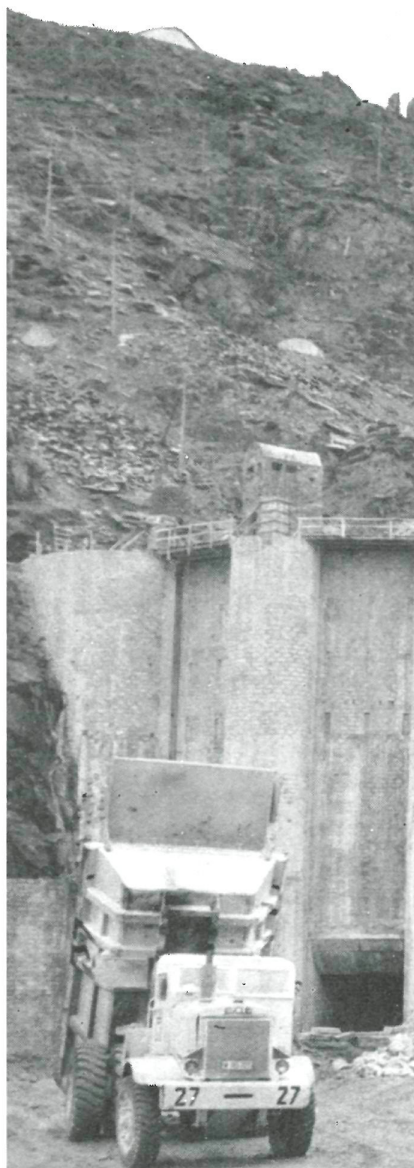
principales y auxiliares de que se disponga.

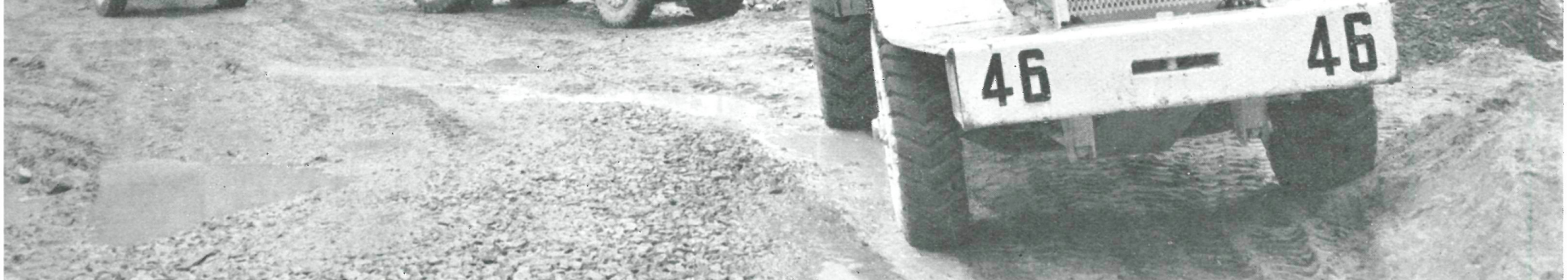




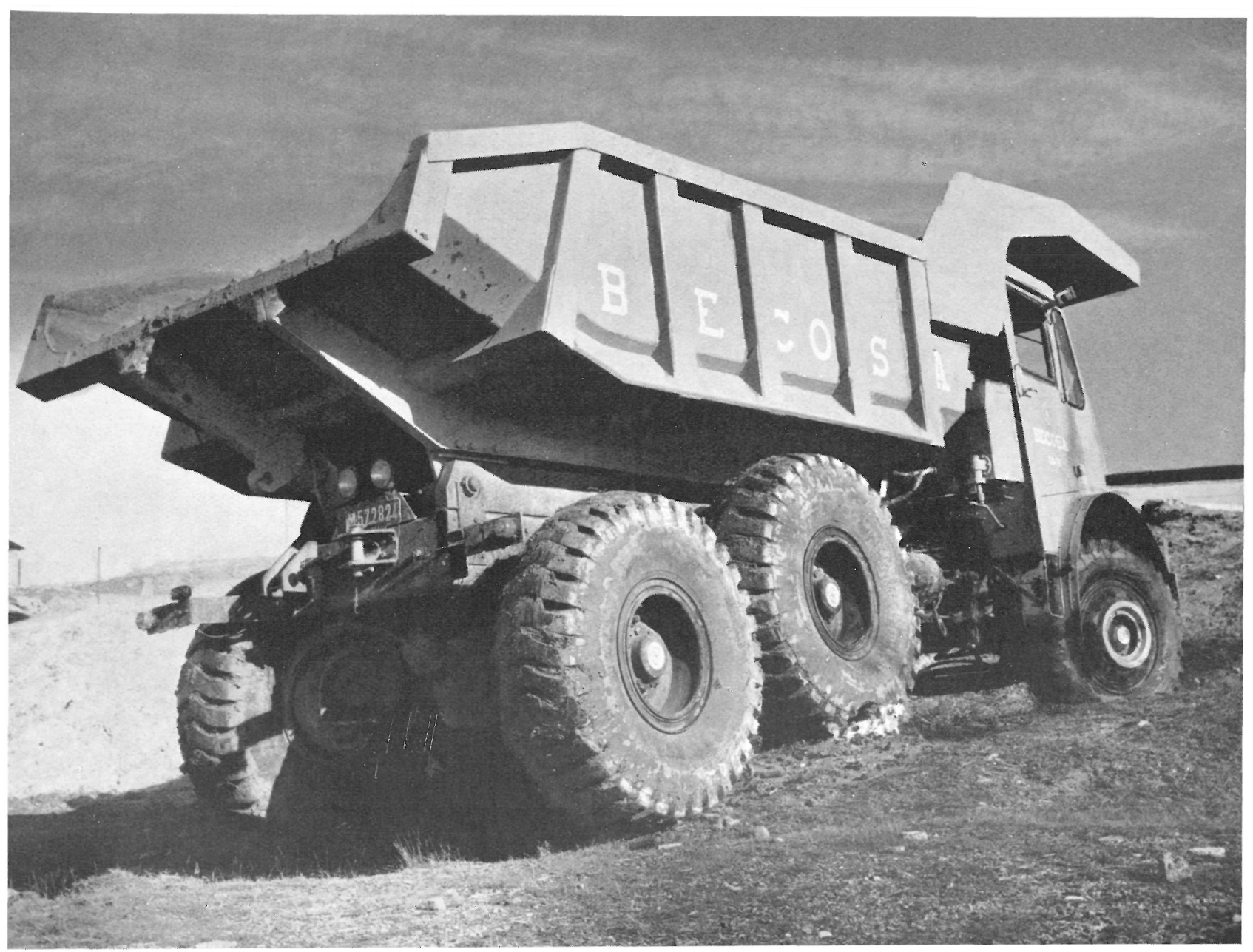

Cuando la distancia entre los puntos de carga y descarga del material está comprendida entre los 500 y $3.500 \mathrm{~m}$, los equipos más adecuados para realizar el transporte son el camión adaptado, o el dumper, dependiendo el empleo de cada uno de ellos de las disponibilidades existentes y las circunstancias especiales y particulares del caso que se trate.

Características económicas.-La inversión precisa para la compra de cada equipo es francamente favorable para la adquisición de camiones; sin embargo, si se examinan los servicios y rendimientos que prestarán cada uno de los dos tipos de vehículos, la complejidad y variabilidad de los diversos factores harán imposible la adopción de una solución óptima para los diversos casos y tipos de trabajos que pueden presentarse.

No puede presupuestarse una cuota de amortización para el camión análoga o deducida de la duración prevista para dicho tipo de vehículo en trabajos y recorridos sobre carreteras. Será preciso considerar reducida la vida y duración del camión y calcular unos gastos suplementarios de entretenimiento y de las pérdidas que se sufrirán por inmovilizaciones, ya que éstos incrementarán sensiblemente los gastos de amortización.

El dumper, construido y concebido para trabajos sobre «todo-terreno», envejecerá más lentamente y su duración puede considerarse superior desde un 30 a un $100 \%$ a la de un camión análogo adaptado para estas misiones.

Para trabajos en los que los recorridos sean efectuados, en su mayor parte, sobre terrenos accidentados y superficies irregulares, el dumper presentará indudables ventajas económicas $\mathrm{y}$, por tanto, se debe prescindir en lo posible del empleo de camiones.

Sin embargo, como las empresas dispondrán de camiones para transportes sobre carretera será preciso y conveniente el empleo de los mismos, aun en trabajos que no les son apropiados. El camión es más económico y su empleo más ventajoso cuando las longitudes de transporte sean superiores a los $3 \mathrm{~km}$, o cuando el terreno sobre el que se efectúen los trabajos ofrezcan unas características que permitan el empleo prolongado de la directa.

Características mecánicas.-Las características diferenciales más acusadas entre ambos tipos de vehículos son:

Aceleración mayor en el dumper y espacios requeridos para la maniobra y el frenado más reducidos.

Mayor movilidad del dumper sobre terrenos blandos y accidentados, debida a su sistema de tracción y al dispositivo de cambio de velocidades que evita la pérdida de tiempo inherente al manejo de la caja de cambios del camión.

Mayor resistencia mecánica del dumper, con la consiguiente disminución del riesgo de averías probables.

Los camiones que se utilizan en estos trabajos deberán estar siempre adaptados al mismo, satisfacer el mayor número de condiciones posibles y ser capaces de desarrollar velocidades del orden de los 80 kilómetros por hora.

Los sistemas de suspensión, transmisión y tracción deberán estar modificados y reforzados de modo que presenten las menores limitaciones posibles para este tipo de trabajos.

El refuerzo del chasis y la solidez de la caja de que haya sido preciso dotarles, no deberán incrementar en exceso el peso muerto o tara del vehículo. 
Ser capaces de soportar cargados inclinaciones laterales del $20 \%$ sin peligro de su estabilidad, poseyendo, además, una máxima altura de franqueo y silueta lo menos acentuada posible.

Poseer motor potente, de alta resistencia mecánica, gran adherencia, dotados de neumáticos de perfil antideslizante, suspensión fuerte, ejes flotantes, mínimo radio de giro y capaces de subir fuertes pendientes con la carga estipulada.

Si la mayoría de los recorridos se van a efectuar sobre terrenos y lugares accidentados, puede establecerse de antemano que el camión sufrirá inmovilizaciones para proceder a su reparación, tanto más numerosas prolongadas y costosas cuanto peor sea la superficie sobre la que han de transitar, con la agravación correspondiente a la tendencia existente de sobrecargar los camiones, todo lo cual conduce a una menor duración de los mismos.

Una solución apropiada puede ser, en determinados casos, el empleo de camiones de chasis corto dotado de sistema de elevación, y caja móvil desmontable del bastidor del camión.

Este sistema permite la colocación de cajas en el suelo para su carga a mano, cosa que se realiza fácilmente dada la escasa altura de la misma sobre el suelo. Una vez llena, es recogida la caja correspondiente por un camión y trasladada al lugar de descarga. Este sistema es, en principio, ventajoso para obras de pequeña envergadura y en las que no se emplee la maquinaria adecuada para la carga de los camiones, siendo la mano de obra económica.

Características técnicas.-Los últimos factores que habrán de estimarse para determinar el tipo de vehículo a utilizar serán:

Los medios disponibles.

La clase de trabajo a realizar.

El tipo medio de terreno sobre el que habrá de efectuarse.

Las características de trabajo y rendimientos que proporcionarán.

La flexibilidad y elasticidad de cada uno, que permitirá la fácil adaptación a las diversas necesidades y situaciones que se irán presentando en el transcurso de la obra.

La rapidez que proporcionará su empleo y la potencia y rendimiento económico de cada uno.

La facilidad de preparación, organización y ejecución del trabajo.

Las limitaciones de empleo.

La especialización del personal disponible para el uso de los vehículos en condiciones de la máxima economía y conservación.

La mayor o menor facilidad de resolución para cada tipo de los problemas de abastecimientos, entretenimiento y circulación.

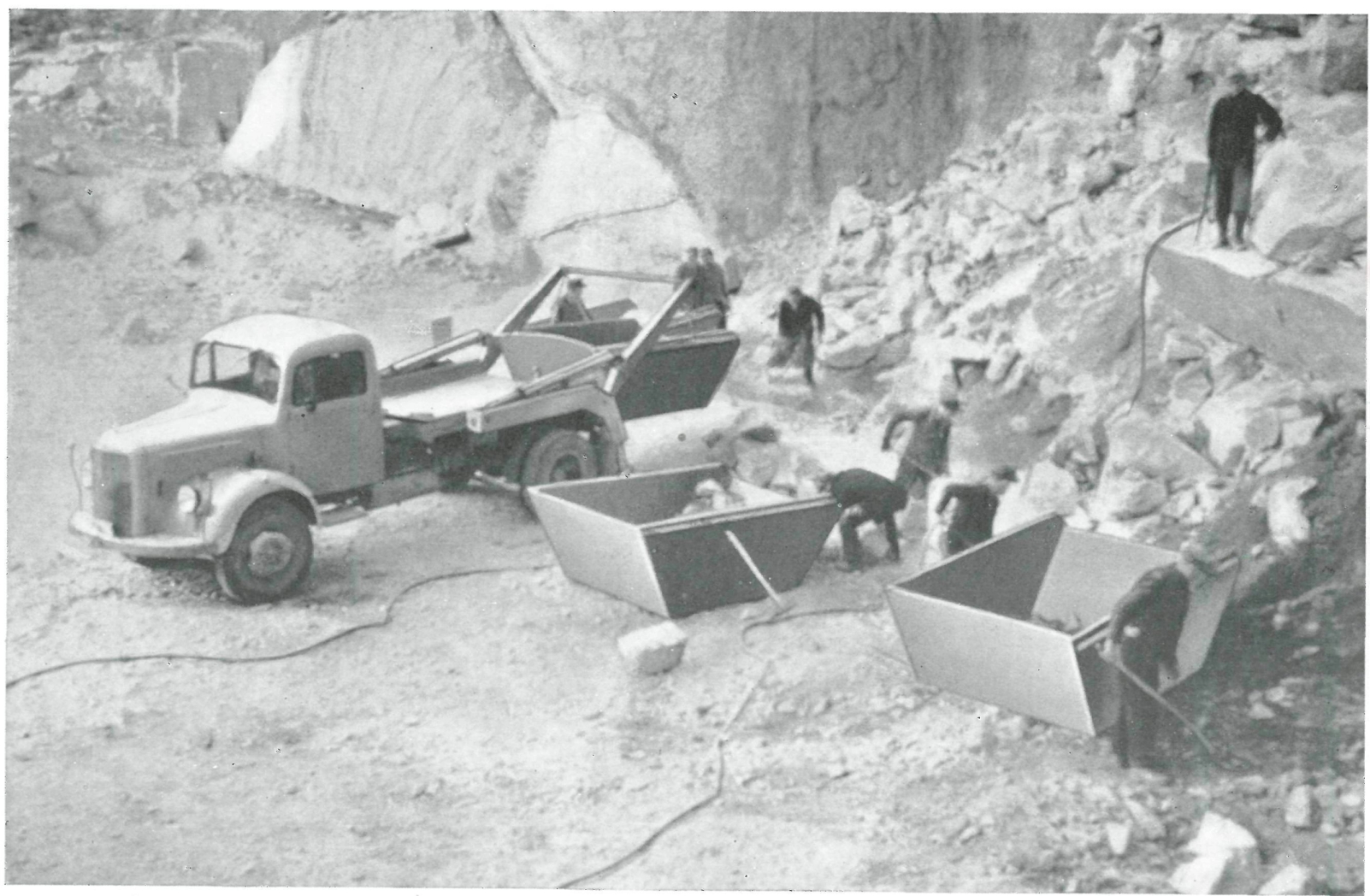




\section{Choix du umogen de trumsport le plus ad áquat pour Ie roulage des matérioux d̀ des distrances mogemmes}

Pour le bon choix du type de machines le plus propre à ces travaux, il faudra tenir compte de quelques caractéristiques économiques, mécaniques et techniques qui fournissent l'information nécessaire pour adopter la solution la plus indiquée.

Parmi les caractéristiques économiques, il faut estimer les coûts d'acquisition et d'entretien, ainsi que les rendements qui seront obtenus en employant chaque type de véhicule.

Parmi les caractéristiques mécaniques, sont considérées celles que présente chacun des types de véhicules, camions, dumpers et camions spéciaux afin de choisir, dans les diverses sortes et les disponibilités existantes, le type qui, pour ses qualités, permette la réalisation normale du travail à exécuter, ainsi que les conditions mécaniques que devront remplir les véhicules adoptés afin de pouvoir concourir, même dans des conditions moins avantageuses, avec ceux qui ont été spécifiquement construits et projetés pour cette sorte de travaux.

Les caractéristiques techniques, dont il faudra tenir compte pour procéder au choix, découlent en réalité des deux caractéristiques précédentes et dépendent des conditions dans lesquelles l'ouvrage sera exécuté, ainsi que des moyens principaux et auxiliaires dont on dispose.

\section{Choice of the most apt mecus of transporting manemials DVer medium distanderes}

To select the appropriate type of equipment the following will have to be taken into account. Economic, mechanical and technical factors and data which indicate the most satisfactory type of vehicle.

Economic factors include purchase price, maintenance, as well as overall performance of each kind of transport.

Mechanical factors are those that characterise each variety of vehicle, such as trucks, dumpers, special lorries. Choice must be directed towards the type most apt to do the required work. Attention must also be given to the mechanical qualities that will enable certain vehicles to compete successfully even in unfavourable conditions against other vehicles which have been specially designed and built to undertake the particular type of work.

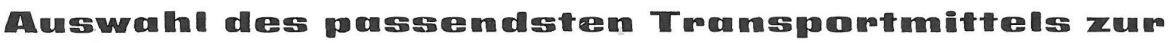

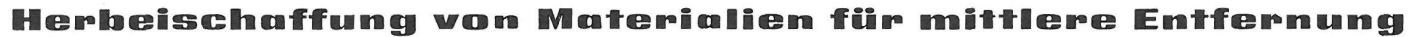

Für die treffende Auswahl der geeignetesten Maschinenart für diese Arbeiten wird man beachten müssen: Einige wirtschaftliche, mechanische und technische Eigenschaften, die die notwendige Auskunft verschaffen, um die geeigneteste Lösung anzunehmen.

Unter den wirtschaftlichen Eigenschaften hat man die Kosten der Anschaffung, Erhaltung und Instandhaltung zu betrachten, sowie die Leistungsfähigkeit, die man mit dem Einsatz ieden Fahrzeuges erreicht.

Unter den mechanischen Eigenschaften versteht man die, die jeder der Fahrzeugarten darstellt, Lastwagen, Dumper und Speziallastwagen, um innerhalb der verschiedenen Arten und bestehenden Verfügbarkeiten die auszuwählen, die durch ihre Eigenschaften die normale Durchführung der auszuführenden Arbeit erlauben, sowie die mechanischen Bedingungen, die die angepassten Fehrzeuge zu erfüllen haben, um befähigt sein zu können, selbst unter weniger vorteilhaften Bedingungen mit denjenigen konkurrieren zu können, die speziell für Arbeiten dieser Art gebaut und geplant wurden.

Die technischen Eigenarten, die bei der Auswahl in Rechnung zu ziehen sind, werden eigentlich von den an deren beiden abgeleitet und sind abhängig von den Bedingungen, unter welchen das Werk durchzuführen ist, sowie von den Haupt-und Hilfsmitteln, über die man verfügt. 